\title{
Quantum reconstruction of an intense polarization squeezed optical state
}

Marquardt, Ch.; Heersink, J.; Dong, R.; Chekhova, M.V.; Klimov, A.B.; Sanchez-Soto, L.L.; Andersen, Ulrik Lund; Leuchs, G.

Published in:

Physical Review Letters

Link to article, DOI:

10.1103/PhysRevLett.99.220401

Publication date:

2007

Document Version

Publisher's PDF, also known as Version of record

Link back to DTU Orbit

Citation (APA):

Marquardt, C., Heersink, J., Dong, R., Chekhova, M. V., Klimov, A. B., Sanchez-Soto, L. L., Andersen, U. L., \& Leuchs, G. (2007). Quantum reconstruction of an intense polarization squeezed optical state. Physical Review Letters, 99(22), 220401. https://doi.org/10.1103/PhysRevLett.99.220401

\section{General rights}

Copyright and moral rights for the publications made accessible in the public portal are retained by the authors and/or other copyright owners and it is a condition of accessing publications that users recognise and abide by the legal requirements associated with these rights.

- Users may download and print one copy of any publication from the public portal for the purpose of private study or research.

- You may not further distribute the material or use it for any profit-making activity or commercial gain

- You may freely distribute the URL identifying the publication in the public portal 


\title{
Quantum Reconstruction of an Intense Polarization Squeezed Optical State
}

\author{
Ch. Marquardt, ${ }^{1}$ J. Heersink, ${ }^{1}$ R. Dong, ${ }^{1}$ M. V. Chekhova, ${ }^{2}$ A. B. Klimov, ${ }^{3}$ L. L. Sánchez-Soto, ${ }^{1}$ \\ U. L. Andersen, ${ }^{1,4}$ and G. Leuchs ${ }^{1}$ \\ ${ }^{1}$ Institute of Optics, Information and Photonics (Max Planck Research Group), University of Erlangen-Nuremberg, \\ Günther-Scharowsky Straße 1, Building 24, 91058 Erlangen, Germany \\ ${ }^{2}$ Department of Physics, M. V. Lomonosov Moscow State University, 119992 Moscow, Russia \\ ${ }^{3}$ Departamento de Física, Universidad de Guadalajara, 44420 Guadalajara, Jalisco, Mexico \\ ${ }^{4}$ Department of Physics, Technical University of Denmark, Building 309, 2800 Lyngby, Denmark
}

(Received 29 January 2007; published 27 November 2007)

\begin{abstract}
We perform a reconstruction of the polarization sector of the density matrix of an intense polarization squeezed beam starting from a complete set of Stokes measurements. By using an appropriate quasidistribution, we map this onto the Poincaré space, providing a full quantum mechanical characterization of the measured polarization state.
\end{abstract}

DOI: 10.1103/PhysRevLett.99.220401

Efficient methods of quantum-state reconstruction are of the greatest relevance for quantum optics. Indeed, they are invaluable for verifying and retrieving information. Since the first theoretical proposals [1] and the pioneering experiments [2], this discipline has witnessed significant growth [3]. Laboratory demonstrations of state tomography are numerous and span a broad range of physical systems, including molecules [4], ions [5], atoms [6], spins [7], and entangled photon pairs [8].

Polarization properties of nonclassical light are becoming more and more important for quantum information applications, since they are simpler to manipulate and can be treated independently of other degrees of freedom. A good example is polarization squeezing, which is detected much more easily than quadrature squeezing. However, to go beyond the standard characterization of polarization squeezing by measuring variances of the Stokes parameters [9], a complete tomography of the state is required. When trying to do this, one is immediately faced with theoretical difficulties: The description of the polarization state in terms of the total density operator is superfluous because it contains not only polarization information. This redundancy can be handled for a low number of photons but becomes a significant hurdle for highly excited states. An adequate solution has been found only recently: It suffices to reconstruct a subset of the density matrix that has been termed the polarization sector [10] (or the polarization density operator [11]), since its knowledge allows for a complete characterization of the polarization state [12].

The purpose of this Letter is to report on the first reconstruction of intense polarization squeezed states. Indeed, our results confirm that, even at this bright limit, they still preserve fingerprints of very strong nonclassical behavior.

We begin by briefly recalling some background material. We assume a two-mode field that is fully described by two complex amplitudes, denoted by $\hat{a}_{H}$ and $\hat{a}_{V}$, where the subscripts $H$ and $V$ indicate horizontal and vertical polar-
PACS numbers: 03.65.Wj, 03.65.Ta, 42.50.Dv, 42.50.Lc

ization modes, respectively. The commutation relations of these operators are standard: $\left[\hat{a}_{j}, \hat{a}_{k}^{\dagger}\right]=\delta_{j k}$, with $j, k \in$ $\{H, V\}$. The analysis of the polarization structure is greatly simplified if we use the Schwinger representation

$$
\begin{gathered}
\hat{J}_{1}=\frac{1}{2}\left(\hat{a}_{H}^{\dagger} \hat{a}_{V}+\hat{a}_{V}^{\dagger} \hat{a}_{H}\right), \quad \hat{J}_{2}=\frac{i}{2}\left(\hat{a}_{H} \hat{a}_{V}^{\dagger}-\hat{a}_{H}^{\dagger} \hat{a}_{V}\right), \\
\hat{J}_{3}=\frac{1}{2}\left(\hat{a}_{H}^{\dagger} \hat{a}_{H}-\hat{a}_{V}^{\dagger} \hat{a}_{V}\right),
\end{gathered}
$$

together with the total photon number $\hat{N}=\hat{a}_{H}^{\dagger} \hat{a}_{H}+\hat{a}_{V}^{\dagger} \hat{a}_{V}$. These operators coincide, up to a factor $1 / 2$, with the Stokes operators, whose average values are precisely the classical Stokes parameters. One immediately finds that $\hat{\mathbf{J}}=\left(\hat{J}_{1}, \hat{J}_{2}, \hat{J}_{3}\right)$ satisfy the commutation relations distinctive of the su(2) algebra: $\left[\hat{J}_{1}, \hat{J}_{2}\right]=i \hat{J}_{3}$ and cyclic permutations. This noncommutability precludes the simultaneous precise measurement of the physical quantities they represent.

The Hilbert space $\mathcal{H}$ for these fields has a convenient orthonormal basis in the form of the Fock states for both polarization modes, namely, $\left|n_{H}, n_{V}\right\rangle$. However, it is advantageous to use the basis $|J, m\rangle$ of common eigenstates of $\hat{J}^{2}$ and $\hat{J}_{3}$. Since $J=N / 2$, this can be accomplished just by relabeling the Fock basis as $|J, m\rangle \equiv$ $\left|n_{H}=J+m, n_{V}=J-m\right\rangle$. Here, for fixed $J$ (i.e., fixed $N), m$ runs from $-J$ to $J$, and these states span a $(2 J+$ 1)-dimensional subspace wherein $\hat{\mathbf{J}}$ acts in the standard way. Since any polarization observable has a blockdiagonal form in this basis, it seems appropriate to define the polarization density operator as

$$
\hat{\varrho}=\bigoplus_{J=0}^{\infty} \hat{\varrho}_{J}=\sum_{J=0}^{\infty} \sum_{m, m^{\prime}=-J}^{J} \varrho_{m m^{\prime}}^{J}|J, m\rangle\left\langle J, m^{\prime}\right| .
$$

For a reconstruction of the quantum state, one first has to extract the required data from tomographical measurements. The overall scheme of our experimental setup is illustrated in Fig. 1. The field to be characterized is ana- 
lyzed by using a general polarization measurement apparatus consisting of a half-wave plate $(\lambda / 2, \theta)$ followed by a quarter-wave plate $(\lambda / 4, \phi)$ and a polarizing beam splitter (PBS). The wave plates transform the input polarization by allowing the measurement of different Stokes parameters by the projection onto the basis $|J, m\rangle$.

The PBS outputs are measured directly by using detectors with InGaAs photodiodes (custom-made by Laser Components $\mathrm{GmbH}$ with $98 \%$ quantum efficiency at dc). The rf currents of the photodetectors are mixed with an electronic local oscillator at $17.5 \mathrm{MHz}$, low-pass filtered $(1.9 \mathrm{MHz})$ to avoid ac saturation due to the laser repetition rate, amplified (FEMTO DHPVA-100), and digitized by an analog-to-digital exit converter (Gage CompuScope 1610) at $10^{7}$ samples per second with a 16-bit resolution and 10 times oversampling. The measurement is limited by the digitally low-pass-filtered resolution bandwidth of $1 \mathrm{MHz}$ at the $17.5 \mathrm{MHz}$ sideband relative to the $200 \mathrm{THz}$ carrier. The signal is sampled around this sideband to avoid the classical noise present in the frequency band around the carrier [13]. Ten digitized samples correspond to the photocurrent at this sideband generated by photons impinging on the photodiode for $1 \mu \mathrm{s}$. This photodetection can be modeled by the positive operator-valued measure $\hat{\Pi}_{m}^{J}=$ $|J, m\rangle\langle J, m|$, so that $w_{m}^{J}=\operatorname{Tr}\left(\hat{\varrho} \hat{\Pi}_{m}^{J}\right)$ is the probability of detecting $n_{H}=J+m$ photons in the horizontal mode and simultaneously $n_{V}=J-m$ photons in the vertical one [14].

Polarization transformations performed by the wave plates can be described in terms of $\hat{J}_{2}$, which generates rotations about the direction of propagation, and $\hat{J}_{3}$, which generates phase shifts between the modes. Their action is represented by $\hat{\mathcal{R}}(\mathbf{n})=e^{i \theta \hat{J}_{2}} e^{i \phi \hat{J}_{3}}$, where $\mathbf{n}=(\cos \phi \sin \theta$, $\sin \phi \sin \theta, \cos \theta)$ is a unit vector given by the angles $(\theta, \phi)$. The experimental histograms recorded for each $\mathbf{n}$ then correspond to the tomographic probabilities

$$
w_{m}^{J}(\mathbf{n})=\operatorname{Tr}\left[\hat{\varrho} \hat{\Pi}_{m}^{J}(\mathbf{n})\right]={ }_{\mathbf{n}}\langle J, m|\hat{\varrho}| J, m\rangle_{\mathbf{n}},
$$

where $\quad \hat{\Pi}_{m}^{J}(\mathbf{n})=\hat{\mathcal{R}}(\mathbf{n}) \hat{\Pi}_{m}^{J} \hat{\mathcal{R}}^{\dagger}(\mathbf{n}) \quad$ and $\quad|J, m\rangle_{\mathbf{n}}=$ $\hat{\mathcal{R}}^{\dagger}(\mathbf{n})|J, m\rangle$.

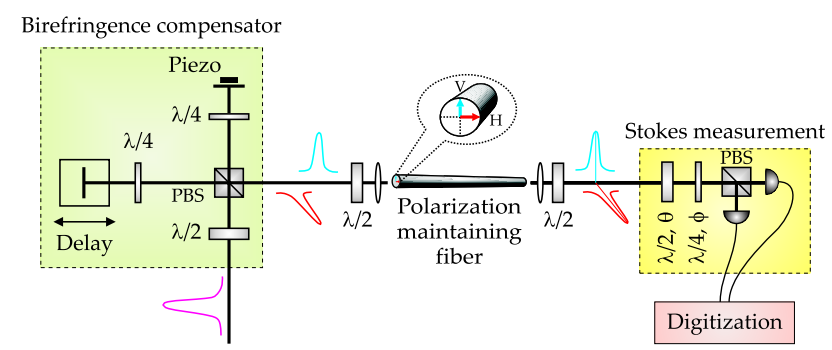

FIG. 1 (color online). Setup for efficient polarization squeezing generation and the corresponding Stokes measurement apparatus.
The quantum states on which we performed the measurements were polarization squeezed states created by the Kerr nonlinearity experienced by ultrashort laser pulses in optical fibers [15]. Our experiment employs a $\mathrm{Cr}^{4+}: \mathrm{YAG}$ laser emitting near Fourier-limited 140 fs FWHM pulses at $1497 \mathrm{~nm}$ with a repetition rate of $163 \mathrm{MHz}$. By using the two polarization axes of a $13.2 \mathrm{~m}$ birefringent fiber (3M FS-PM-7811, $5.6 \mu \mathrm{m}$ mode-field diameter), two quadrature squeezed states are simultaneously and independently generated. The emerging pulses intensities are set to be identical, and they are aligned to temporally overlap. The fiber polarization axes exhibit a strong birefringence (beat length of $1.67 \mathrm{~mm}$ ) that must be compensated. To minimize postfiber losses, we precompensate the pulses in an unbalanced Michelson-like interferometer that introduces a tunable delay between the polarizations [16]. A small part $(0.1 \%)$ of the fiber output serves as the input to a control loop to maintain a $\pi / 2$ relative phase between the exiting pulses, producing a circularly polarized beam.

Since the Kerr effect is photon-number-preserving, the amplitude fluctuations of the two individual modes $H$ and $V$ are at the shot-noise level. This was checked by using a coherent beam from the laser and employing balanced detection. The average output power from the fiber was $13 \mathrm{~mW}$, which, with the bandwidth definition of our quantum state, corresponds to an average number of photons of $10^{11}$ per $1 \mu \mathrm{s}$. The Kerr effect in fused silica generates squeezing up to some terahertz, making the choice of the sideband, in principle, arbitrary. Rotation of the wave

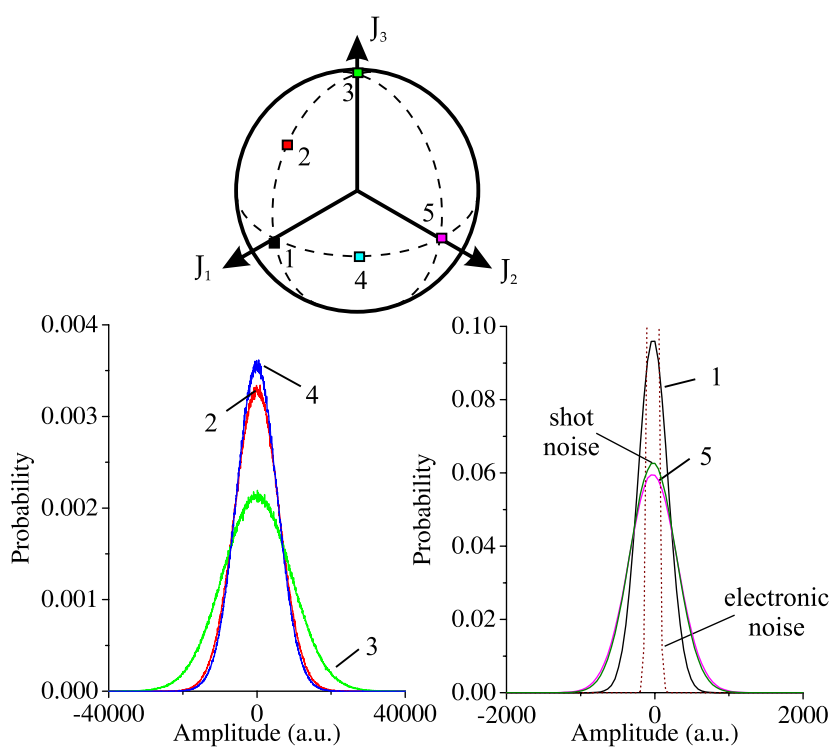

FIG. 2 (color online). Measured histograms of the difference current of the two detectors for various measurement angles on the Poincare sphere. When the total number of photons is not measured, the histograms are the sum over $J$ of the tomograms (3). Note the different scales on both plots. Histograms 1, 2, and 3 are in the dark plane, while histogram 5 is at the classical mean value. The histograms corresponding to electronic and shot noise are also shown. 
plates scanned one-quarter of the Poincare sphere in 65 steps for $\phi$ and 64 steps for $\theta$, a measurement which took over five hours. The rest of the data can be deduced from symmetry.

For each pair of angles, the photocurrent noise of both detectors after the PBS was simultaneously sampled $2.9 \times 10^{6}$ times. Noise statistics of the difference of the two photocurrents were acquired in histograms with 2048 bins and the optical intensities incident on both detectors were recorded as well (as dc current values). In Fig. 2, we show typical histograms at different angles on the Poincare sphere. As the widths of the histograms largely vary from squeezing to antisqueezing ranges, there are two plots with the amplitude scale differing by more than 1 order of magnitude. The histograms labeled 1-3 are measured in the dark plane, which is perpendicular to the classical mean value of the state. Label 1 denotes the angle of maximum squeezing, while label 3 corresponds to the antisqueezing. Label 5 is the angle of the classical mean value, where the measured noise data are almost shot-noise limited. Because of the high number of samples, the measured histograms are smooth, and, at the same time, the number of bins makes it possible to resolve the large dynamical range of amplitudes, so no data interpolation was needed. We also plot histograms showing the electronic noise and the shot noise. Higher-order moments of the measured data were also computed, but we found no significant deviation from what is expected from a Gaussian distribution.
The reconstruction in each $(2 J+1)$-dimensional invariant subspace can be now carried out exactly since it is essentially equivalent to a spin $J$ [17]. After some calculations, one finds that

$$
\hat{\varrho}_{J}=\frac{1}{4 \pi} \sum_{m=-J}^{J} \int_{\mathcal{S}_{2}} d \mathbf{n}^{\prime} w_{m}^{J}\left(\mathbf{n}^{\prime}\right) \mathcal{K}_{J}\left(m-\mathbf{n}^{\prime} \cdot \hat{\mathbf{J}}\right),
$$

where the integration extends over the unit sphere $\mathcal{S}_{2}$ and the kernel $\mathcal{K}_{J}(x)$ is

$$
\mathcal{K}_{J}(x)=\frac{2 J+1}{4 \pi^{2}} \int_{0}^{2 \pi} d \omega \sin ^{2}\left(\frac{\omega}{2}\right) e^{-i \omega x} .
$$

From the exact solution (4), one can calculate any polarization quasidistribution [18]. From a computational perspective, the $\mathrm{SU}(2) Q$ function turns out to be the simplest, since in each invariant subspace it reduces to

$$
Q(J, \mathbf{n})=\left\langle J, \mathbf{n}\left|\hat{\varrho}_{J}\right| J, \mathbf{n}\right\rangle,
$$

where $|J, \mathbf{n}\rangle=\hat{\mathcal{R}}(\mathbf{n})|J, m=-J\rangle$ are $\mathrm{SU}(2)$ coherent states obtained by displacing the "ground" state $|J,-J\rangle$ over the sphere [19]. This definition is a straightforward generalization of the standard one for the harmonic oscillator. The Wigner function can also be evaluated, although with additional effort. Nevertheless, we do not expect these two quasidistributions to differ notably for the states we study here. We need only thus to calculate the matrix elements of the kernel $\mathcal{K}_{J}\left(m-\mathbf{n}^{\prime} \cdot \hat{\mathbf{J}}\right)$. The most direct way to proceed is to note that

$$
\left\langle J, \mathbf{n}\left|\mathcal{K}_{J}\left(m-\mathbf{n}^{\prime} \cdot \hat{\mathbf{J}}\right)\right| J, \mathbf{n}\right\rangle=\frac{2 J+1}{4 \pi^{2}} \int_{0}^{2 \pi} d \omega \sin ^{2}\left(\frac{\omega}{2}\right) e^{i m \omega}\left[\cos \left(\frac{\omega}{2}\right)-i \sin \left(\frac{\omega}{2}\right) \cos \chi\right]^{2 J},
$$

where $\cos \chi=\mathbf{n} \cdot \mathbf{n}^{\prime}$. In the limit of $J \gg 1$, the integral in Eq. (7) reduces to $d^{2} \delta(x) / d x^{2}$ evaluated at $x=m-J \mathbf{n} \cdot \mathbf{n}^{\prime}$. Since $m$ can be taken as a quasicontinuous variable, we integrate by parts to obtain

$$
Q(J, \mathbf{n})=\frac{2 J+1}{4 \pi^{2}} \int_{-\infty}^{\infty} d m \int_{\mathcal{S}_{2}} d \mathbf{n}^{\prime} \frac{d^{2} w_{m}^{J}(\mathbf{n})}{d m^{2}} \delta\left(m-J \mathbf{n} \cdot \mathbf{n}^{\prime}\right) .
$$

Thus, in the limit of high photon numbers, the reconstruction turns out to be equivalent to an inverse Radon transform [20] of the measured tomograms, which greatly simplifies the numerical evaluation of $Q(J, \mathbf{n})$.

In Fig. 3 (top), we show the result of the threedimensional inverse Radon transform for a polarization squeezed state. Here an isocontour surface of $Q(J, \mathbf{n})$ in the Poincare space (that results from representing the average values of $\hat{\mathbf{J}}$ in a three-dimensional Euclidean space having $J_{1}, J_{2}$, and $J_{3}$ as orthogonal axes) is seen. The ellipsoidal shape of the state is clearly visible. The antisqueezed direction of the ellipsoid is dominated by excess noise stemming largely from photon-phonon interactions, which is characteristic of squeezed states generated in optical fibers.

In Fig. 3 (bottom), we compare the projections on the coordinate planes of the isocontour surfaces of a coherent and a polarization squeezed state for the value correspond- ing to the half maximum. The contours agree with the $6.2 \pm 0.3 \mathrm{~dB}$ squeezing that was directly measured with a spectrum analyzer. The elliptical contour in the $J_{1}-J_{3}$

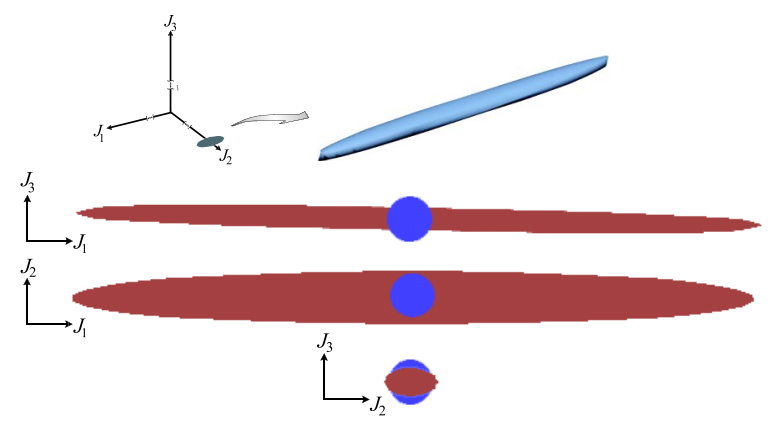

FIG. 3 (color online). Sections of the isocontour surface plots of the $Q$ function for a coherent state (blue) and a polarization squeezed state (red). 


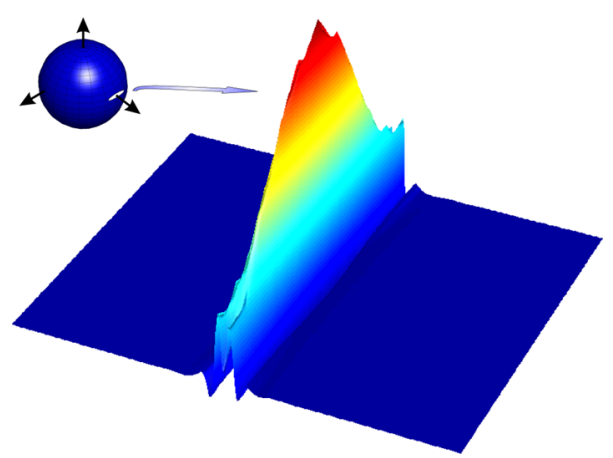

FIG. 4 (color online). Probability distribution $Q(\mathbf{n})$ over the Poincaré unit sphere for a polarization squeezed state obtained over all invariant subspaces. The distribution is strongly concentrated at the classical mean value, so we show a zoomed version.

plane is slightly rotated, which is related to the squeezing angle [15]. The projections on the planes $J_{1}-J_{2}$ and $J_{2}-J_{3}$ show an additional spreading of the squeezed state in the $J_{2}$ direction caused by the imperfect polarization contrast in the measurement setup that mixes some of the antisqueezing in the $J_{2}$ direction. As the classical excitation of the state is in the $J_{2}$ direction, one expects to reach the shotnoise limit in this projection.

By summing over all of the values of $J$, we obtain the total $Q(\mathbf{n})$, which is a probability distribution over the Poincaré unit sphere and is properly normalized. In Fig. 4, we have plotted such a function for the squeezed state. As the state has a large excitation and the angles of the distribution on the unit sphere are small, the spherical coordinates can be treated as Cartesian in the vicinity of the classical point, and we present a zoomed version of the surface of the sphere. Again the excess phase noise is visible. We have checked that the sampling errors affect only the background noise by less than $\pm 1 \%$ compared to the maximum value of the distribution. The observed negative values of the $Q$ function near the main peak (about 9\% of the maximum) are unphysical: They arise from the inverse Radon transformation and have a twofold origin. First, the extremely elongated structure creates oscillations in the reconstructed distribution, which has been confirmed by numerical simulations. The second source is the laser, which had occasional power spikes of $5 \%$ and an overall power drift of $8 \%$ over the measurement time. This power drift led to a corresponding drift of the state, and, as a result, various tomograms relate to slightly different states.

We stress that our setup gives only information about the polarization sector. While there are other proposals to reconstruct the whole density matrix of a two-mode field [21], those schemes employ homodyne detection in both modes, which is unattainable in our case due to the high intensities of our states.

In summary, we have presented a full quantum reconstruction of an intense polarization squeezed state created by the Kerr effect in an optical fiber. Interesting future investigations include a comparison with maximum likelihood methods and a reconstruction of nonclassical polarization states with lower intensity.

We thank V.P. Karassiov for useful discussions and C. Müller for technical assistance. Financial support from the EU (COVAQIAL No. FP6-511004), CONACyT (Grant No. 45704), and DGI (Grant No. FIS2005-0671) is gratefully acknowledged. M. V.C. was supported by DFG (Grants No. 436 RUS 17-75-05 and No. 436 RUS 17-7606).

[1] R. G. Newton and B. L. Young, Ann. Phys. (N.Y.) 49, 393 (1968); W. Band and J.L. Park, Found. Phys. 1, 133 (1970); J. Bertrand and P. Bertrand, ibid. 17, 397 (1987); K. Vogel and H. Risken, Phys. Rev. A 40, 2847 (1989).

[2] D. T. Smithey et al., Phys. Rev. Lett. 70, 1244 (1993).

[3] U. Leonhardt, Measuring the Quantum State of Light (Cambridge University Press, Cambridge, England, 1997); G. M. D' Ariano, M. G. A. Paris, and M.F. Sacchi, in Advances in Imaging and Electron Physics Vol. 128, edited by P. Hawkes (Elsevier, New York, 2003), pp. 205-308; Quantum State Estimation, edited by M. G. A. Paris and J. Řeháček, Lect. Notes Phys. (Springer, Heidelberg, 2004), Vol. 649; A. I. Lvovsky and M. G. Raymer, arXiv:quant-ph/0511044.

[4] T. J. Dunn, I. A. Walmsley, and S. Mukamel, Phys. Rev. Lett. 74, 884 (1995).

[5] D. Leibfried et al., Phys. Rev. Lett. 77, 4281 (1996).

[6] C. Kurtsiefer, T. Pfau, and J. Mlynek, Nature (London) 386, 150 (1997).

[7] I. L. Chuang, N. Gershenfeld, and M. Kubinec, Phys. Rev. Lett. 80, 3408 (1998).

[8] A. G. White et al., Phys. Rev. Lett. 83, 3103 (1999).

[9] W. P. Bowen et al., Phys. Rev. Lett. 88, 093601 (2002).

[10] M. G. Raymer, D. F. McAlister, and A. Funk, in Quantum Communication, Computing, and Measurement 2, edited by P. Kumar (Plenum, New York, 2000), p. 147-162.

[11] V. P. Karassiov, J. Russ. Laser Res. 26, 484 (2005).

[12] P. A. Bushev et al., Opt. Spectrosc. 91, 526 (2001).

[13] S. Schiller et al., Phys. Rev. Lett. 77, 2933 (1996).

[14] A. Agliati et al., J. Opt. B 7, S652 (2005).

[15] J. Heersink et al., Opt. Lett. 30, 1192 (2005).

[16] J. Heersink et al., Phys. Rev. A 68, 013815 (2003).

[17] U. Leonhardt, Phys. Rev. Lett. 74, 4101 (1995); C. Brif and A. Mann, Phys. Rev. A 59, 971 (1999); J. P. Amiet and S. Weigert, J. Phys. A 32, L269 (1999); G. M. D’ Ariano, L. Maccone, and M. Paini, J. Opt. B 5, 77 (2003).

[18] G. S. Agarwal, Phys. Rev. A 24, 2889 (1981); J.P. Dowling, G. S. Agarwal, and W.P. Schleich, ibid. 49, 4101 (1994).

[19] A. Perelomov, Generalized Coherent States (Springer, Berlin, 1986).

[20] S.R. Deans, The Radon Transform and Some of its Applications (Wiley, New York, 1983).

[21] M. G. Raymer and A.C. Funk, Phys. Rev. A 61, 015801 (1999). 